\title{
Morfologia dos esporos das espécies de Leucobryaceae Schimp. (Bryophyta) do Parque Estadual de Ilha Grande, Município de Angra dos Reis, Estado do Rio de Janeiro ${ }^{1}$
}

\author{
ISABELA CRESPO CALDEIRA ${ }^{2,4}$, VANIA GONÇALVES LOURENÇO ESTEVES² e \\ ANDRÉA PEREIRA LUIZI-PONZO ${ }^{3}$
}

(recebido: 2 de junho de 2005; aceito: 1 de junho de 2006)

\begin{abstract}
Spore morphology of Leucobryaceae Schimp. (Bryophyta) species in the Parque Estadual de Ilha Grande, Angra dos Reis, Rio de Janeiro State). The present study aimed to analyze the spore morphology of Leucobryaceae species occurring in the Parque Estadual da Ilha Grande, Ilha Grande Municipality, Rio de Janeiro State, Brazil. Spores of six species distributed in two genera, were analyzed: Leucobryum albicans Schwaegr., L. clavatum Hampe, L. crispum C. Muell., L. martianum (Hornsch.) Hampe ex C. Muell., Octoblepharum albidum Hedw. and O. cocuiense Mitt. Different methods were utilized for spore morphology analysis. The analyzed species presented spore in monads, small to medium sized (10.0-22.5 $\mu \mathrm{m})$, heteropolar, subcircular, with proximal apertural region and granulated surface. The apertural region is irregularly shaped, elongated, subcircular, subtriangular or without defined configuration. The variations detected, and utilized for species separation, are related to spores size and ornamentation element distribution.
\end{abstract}

Key words - Brazil, Leucobryaceae, spore

RESUMO - (Morfologia dos esporos das espécies de Leucobryaceae Schimp. (Bryophyta) do Parque Estadual de Ilha Grande, Município de Angra dos Reis, Estado do Rio de Janeiro). O presente trabalho trata da análise morfológica dos esporos das espécies de Leucobryaceae Schimp. ocorrentes no Parque Estadual da Ilha Grande, Município de Ilha Grande, Rio de Janeiro, Brasil. Foram analisados os esporos de seis espécies distribuídas em dois gêneros: Leucobryum albicans Schwaegr., L. clavatum Hampe, L. crispum C. Muell., L. martianum (Hornsch.) Hampe ex C. Muell., Octoblepharum albidum Hedw. e O. cocuiense Mitt. Diferentes métodos foram utilizados para a análise da morfologia dos esporos. As espécies analisadas apresentaram esporos em mônades, de tamanho pequeno a médio $(10,0-22,5 \mu \mathrm{m})$, heteropolares, subcirculares, com região apertural proximal, superfície granulada. A região apertural é irregular, apresentando-se de forma alongada, subcircular, subtriangular ou sem forma definida. As variações encontradas, e que foram utilizadas para a separação das espécies, estão relacionadas ao tamanho dos esporos e à distribuição dos elementos de ornamentação.

Palavras-chave - Brasil, esporo, Leucobryaceae

\section{Introdução}

A família Leucobryaceae apresenta filídios frágeis, eretos, algumas vezes flexuosos ou curvados, lanceolados a ligulados, com costa espessa, que ocupa a maior parte do filídio; peristômio simples ou ausente, com dentes lanceolados e bífidos, caliptra mitrada ou cuculada e nua.

1. Parte da dissertação de mestrado do primeiro autor, Programa de Pós-Graduação em Ciências Biológicas (Botânica) do Museu Nacional/UFRJ.

2. Museu Nacional/UFRJ, Departamento de Botânica, Quinta da Boa Vista, São Cristóvão 20940-040 Rio de Janeiro, RJ, Brasil.

3. Universidade Federal de Juiz de Fora, Departamento de Botânica, 36036-330 Juiz de Fora, MG, Brasil.

4. Autor para correspondência: belade@terra.com.br
Mueller (1843) sugeriu o nome Leucobryaceae em concordância com o principal gênero Leucobryum Hampe. Entretanto, o nome não foi formalizado com diagnose latina. Schimper (1836-1855) publicou, então, um trabalho no qual descrevia a família Leucobryaceae e a classificava como a família XI dos Musci.

No trabalho de delimitação dos grupos de Musgos, Brotherus (1925) definiu a família Leucobryaceae com nove gêneros.

No Brasil, a família Leucobryaceae está representada por três gêneros, reunidos em 32 espécies (Yano 1996). Na região de estudo do presente trabalho foram registradas seis espécies, pertencentes a dois gêneros.

A Ilha Grande, situada no Município de Angra dos Reis, Rio de Janeiro, está inserida na zona de domínio ecológico da Mata Atlântica, compreendida na zona tropical de clima marítimo com alta pluviosidade. Recentemente, Oliveira-e-Silva \& Yano (2000) 
estudaram os musgos ocorrentes nessa região e notaram o elevado número de espécies de Leucobryaceae com esporófito, o que permitiu o desenvolvimento do presente trabalho.

Estudos acerca da morfologia dos esporos de Bryophyta têm se intensificado nos últimos anos (Sorsa \& Koponen 1973, Boros \& Járai-Komlódi 1975, Hirohama 1977, Olensen \& Mogensen 1978, Brown \& Lemmon 1980, 1984a, b, 1988, 1991, Mogensen 1981, Castañeda Peña 1985, Frahm \& Gradstein 1991, LuiziPonzo \& Barth 1998, 1999) mas, ainda, são necessários estudos adicionais. Com os resultados obtidos no presente estudo, pretende-se auxiliar na definição e caracterização taxonômica das espécies estudadas e oferecer subsídios à identificação de esporos fossilizados em sedimentos terrestres.

No Brasil, trabalhos relacionados à palinologia de espécies da família Leucobryaceae são escassos, LuiziPonzo et al. (1997) estudaram os esporos de uma variedade de Octoblepharum albidum Hedw., ocorrente no Pará. Estudos concernentes às demais espécies não foram relatados na literatura consultada.

Segundo Yano (1975), várias classificações foram elaboradas dentro dessa família desde sua criação e, mais recentemente, Buck \& Goffinet (2000) sugeriram uma reorganização da circunscrição da família, ainda debatida entre os pesquisadores, representando assim, um grupo polêmico quanto à delimitação dos seus gêneros. No presente estudo, foi seguida a classificação de Vitt (1984).

\section{Material e métodos}

Foram estudadas todas as espécies da família Leucobryaceae ocorrentes na Ilha Grande num total de seis espécies. Os critérios para a seleção dos exemplares foram a presença do esporófito e o material ser procedente do Parque Estadual da Ilha Grande.

As exsicatas estudadas foram cedidas pelo Herbário da Universidade do Estado do Rio de Janeiro (HRJ), visto que o maior número de espécimes da família em estudo se encontra depositada neste herbário.

Para cada espécie foram estudados, sempre que possível, os esporos de quatro espécimes para a confirmação dos dados, sendo um destes espécimes utilizado como material padrão para as descrições e ilustrações e os demais, como materiais de comparação. Na listagem de material examinado, os espécimes "padrão" estão assinalados com um asterisco.

Material examinado: Leucobryum albicans (Schwagr.) Lindb.- BRASIL. RIO DE JANEIRO: Angra dos Reis, Ilha Grande, 6-XI-1993, D.P. Costa 1911 (HRJ*), 12-VII-1994, M.I.M.N.
Oliveira-e-Silva 1909 (HRJ), 21-III-1995, O. Yano \& M.I.M.N. Oliveira-e-Silva 23669 (HRJ), 12-VII-1995, M.I.M.N. Oliveira e Silva 1951 (HRJ). Leucobryum clavatum Hampe - BRASIL. Rio De Janeiro: Angra dos Reis, Ilha Grande, 23-X-1994, M.I.M.N. Oliveira-e-Silva 2388 (HRJ*), 24-X-1994, M.I.M.N. Oliveira-e-Silva 2549 (HRJ), 21-III-1995, M.I.M.N. Oliveirae-Silva 3126 (HRJ), 22-III-1995, O. Yano \& M.I.M.N. Oliveirae-Silva 23802 (HRJ). Leucobryum crispum C. Muell. BRASIL. Rio De Janeiro: Angra do Reis, Ilha Grande, 12-VII-1994, O. Yano \& M.I.M.N. Oliveira-e-Silva 1912 (HRJ), 12-VII-1994, O. Yano \& M.I.M.N. Oliveira-e-Silva 2912 (HRJ*), 23-VIII-1994, M.I.M.N. Oliveira-e-Silva 2042 (HRJ), 23-VIII-1994, M.I.M.N. Oliveira-e-Silva 2040 (HRJ), 23-VIII-1994, M.I.M.N. Oliveira-e-Silva 2045 (HRJ), 21-II1995, M.I.M.N. Oliveira-e-Silva 2887 (HRJ). Leucobryum martianum (Hornsch.) Hampe ex C. Muell. - BRASIL. RIo DE JANEIRO: Angra dos Reis, Ilha Grande, 12-VII-1994, M.I.M.N. Oliveira-e-Silva 1898 (HRJ), 12-VII-1994, M.I.M.N. Oliveirae-Silva 1904 (HRJ), 29-XI-1994, M.I.M.N. Oliveira-e-Silva 2493 (HRJ), 21-III-1995, O. Yano \& M.I.M.N. Oliveira-e-Silva 3092 (HRJ*). Octoblepharum albidum Hedw. - BRASIL. RIo DE JANEIRO: Angra dos Reis, Ilha Grande, 15-VI-1994, M.I.M.N. Oliveira-e-Silva 1711 (HRJ), 22-VIII-1994, M.I.M.N. Oliveirae-Silva 2081 (HRJ), 20-XII-1994, M.I.M.N. Oliveira-e-Silva 2606 (HRJ), 10-I-1995, M.I.M.N. Oliveira-e-Silva 2703 (HRJ), 18-IV-1995, M.I.M.N. Oliveira-e-Silva 4112 (HRJ*). Octoblepharum cocuiense Mitt. - BRASIL: RIO DE JANEIRO: Angra do Reis, Ilha Grande, 23-VIII-1994, M.I.M.N. Oliveirae-Silva 2087 (HRJ); Mangaratiba, 14-IX-1993, M.I.M.N. Oliveira e Silva 625 (HRJ*), 26-V-1994, M.I.M.N. Oliveira-eSilva 1557 (HRJ).

Os esporos foram analisados tanto em microscópio de luz (ML), quanto em microscópio eletrônico de varredura (MEV).

Os esporos coletados foram submetidos ao tratamento pelo método de Wodehouse (1935), modificado por LuiziPonzo (2001) com o objetivo de fazer uma avaliação morfológica externa da parede, análise do conteúdo celular, variação do tamanho dos esporos, bem como determinar a heteropolaridade e o estado de maturação. Durante esse tratamento, para cada lâmina, foram selecionados esporos de uma mesma cápsula para evitar grandes variações no estado de maturação dos esporos.

Para a análise da estrutura e ornamentação, bem como para tomada de medidas de tamanho dos esporos e da espessura do esporoderma foi utilizado o método de acetólise (Erdtman 1952). Alguns esporos não resistiram ao processo e foi necessário diminuir o tempo de permanência no banhomaria para um minuto, bem como diminuir a temperatura para $80^{\circ} \mathrm{C}$.

Para esporos de grão de pólen cuja polaridade é definida, são efetuadas as medidas dos diâmetros em vista polar e equatorial (Luizi-Ponzo \& Barth 1999, Esteves \& Melhem 2000); no caso da família Leucobryaceae, o pequeno tamanho dos esporos da maioria das espécies gerou dificuldade em 
definir as suas posições nas lâminas, por isso estabeleceuse o diâmetro maior e menor como a medida a ser tomada em todos os materiais selecionados (Mogensen 1981, Luizi-Ponzo 2001).

A avaliação quantitativa dos diâmetros maior e menor se baseou na tomada de medidas de 50 esporos do material padrão, em um mínimo de quatro lâminas. Esses dados foram submetidos à análise estatística, permitindo a apresentação da média aritmética (0), dos limites da sua amplitude $\left(\mathrm{x}_{\min }-\mathrm{x}_{\text {máx }}\right)$, do desvio padrão da média $\left(s_{0}\right)$ do desvio padrão $(s)$, do coeficiente de variação $(C V)$ e do intervalo de confiança a 95\% (IC) na tabela 1.

Para os espécimes denominados “de comparação”, foram medidos 30 esporos, contidos em um mínimo de três lâminas, apresentando-se a média de tamanho (0) e os limites da sua amplitude $\left(\mathrm{x}_{\text {min }}-\mathrm{x}_{\text {máx }}\right)$ na tabela 2.

As médias de tamanho da espessura do esporoderma (E) foram obtidas a partir de 10 esporos, considerando-se perina e exina em conjunto.

A terminologia adotada foi baseada em Barth \& Melhem (1988) e Punt et al. (1999).

\section{Resultados e Discussão}

Os esporos estudados são isomórficos, de tamanho pequeno ou médio (Leucobryum crispum C. Muell.) (tabela 1). Todos os esporos apresentam esporoderma formado por perina, exina e intina (figuras 1-26). A exina se apresenta psilada em todas as espécies, a perina é formada por grânulos com padrões variados de distribuição. Análises realizadas pelo método de Wodehouse (1935) demonstram que a perina apresenta granulações e é mais espessa no pólo proximal.

Os esporos apresentam uma região apertural proximal, confirmando a heteropolaridade dos mesmos.
Essa região mostra variações no contorno, podendo ser alongada (figura 5), subcircular (figuras 14, 21), subtriangular (figuras 10, 11, 25) ou sem forma definida (figura 16).

Leucobryum albicans Schwaegr. (figuras 1-5) Esporos de tamanho pequeno (tabela 1), perina formada por grânulos organizados em muros (figuras 4, 5), distribuídos irregularmente pelo esporoderma. Região apertural alongada (figura 5). Observa-se uma diminuição da quantidade de elementos de ornamentação nesta região.

Leucobryum clavatum Hampe (figuras 6-11) -Esporos de tamanho pequeno (tabela 1), perina formada por grânulos bastante unidos, resultando em uma ornamentação uniforme (figuras 6-9). Região apertural subtriangular, nitidamente delimitada por margem (figuras 10-11).

Leucobryum crispum C. Muell. (figuras 12-15) - Esporos de tamanho pequeno a médio (tabela 1), perina formada por nanogrânulos e grânulos (figuras 12-15). Os nanogrânulos se apresentam isolados ou agrupados, distribuídos irregularmente pela superfície (figura 15). A região apertural é subcircular (figura 14).

Leucobryum martianum (Hornsch.) Hampe ex C. Muell. (figuras 16-17) - Esporos de tamanho pequeno (tabela 1), perina formada por nanogrânulos e grânulos (figura 17). Grânulos presentes na superfície, como resultado da associação dos nanogrânulos, distribuídos irregularmente. Região apertural sem forma definida. Octoblepharum albidum Hedw. (figuras 18-22) - Esporos de tamanho pequeno (tabela 1) perina formada por nanogrânulos e grânulos dispersos irregularmente (figura 20). Os nanogrânulos podem estar unidos, resultando num padrão granulado (figura 20). Região

Tabela 1. Dados morfométricos dos esporos acetolisados do material padrão, em mm (diâmetros maior e menor, $n=50$ e estratos do esporoderma, $n=10$ ). ( $\bar{x}=$ média aritmética; $s_{\bar{x}}=$ desvio padrão da média; $s=$ desvio padrão; $C V=$ coeficiente de variação; $I C=$ intervalo de confiança; $\mathrm{E}=$ espessura do esporoderma).

Table 1. Morphometric data of acetolyzed spores from the reference specimen, in mm (largest and smallest diameter, $n=50$ and sporoderm strata, $n=10)$. ( $\bar{x}=$ arithmetic mean; $s_{\overline{\bar{x}}}=$ average standard deviation; $s=$ standard deviation; $C V=$ coefficient of variation; $I C=$ confidence interval; $\mathrm{E}=$ thickeness of sporoderm).

\begin{tabular}{|c|c|c|c|c|c|c|c|c|c|c|c|}
\hline \multirow[t]{2}{*}{ Táxons/Medidas } & \multicolumn{5}{|c|}{ Diâmetro maior } & \multicolumn{5}{|c|}{ Diâmetro menor } & \multirow[t]{2}{*}{$\mathrm{E}$} \\
\hline & $\mathrm{X}_{\min }-\mathrm{X}_{\operatorname{máx}}$ & $\pm s_{\bar{x}}$ & $s$ & IC & $C V \%$ & $\mathrm{X}_{\min }-\mathrm{X}_{\text {máx }}$ & $\pm S_{\bar{x}}$ & $s$ & IC & $C V \%$ & \\
\hline Leucobryum albicans & $12,5-17,5$ & $15,6 \pm 0,2$ & 1,6 & $15,2-16,0$ & 10,0 & $10,0-15,0$ & $13,2 \pm 0,2$ & 1,7 & $12,8-13,7$ & 12,6 & 2,0 \\
\hline L. clavatum & $15,0-20,0$ & $16,8 \pm 0,2$ & 1,7 & $16,3-17,3$ & 10,1 & $10,0-15,0$ & $13,2 \pm 0,3$ & 1,9 & $12,6-13,8$ & 14,2 & 1,4 \\
\hline L. crispum & $20,0-25,0$ & $23,1 \pm 0,2$ & 1,5 & $22,7-23,5$ & 6,4 & $17,5-22,5$ & $20,6 \pm 0,2$ & 1,5 & $20,2-21,0$ & 7,5 & 2,2 \\
\hline L. martianum & $17,5-20,0$ & $19,0 \pm 0,2$ & 1,3 & $18,6-19,4$ & 7,1 & $15,0-17,5$ & $15,7 \pm 0,2$ & 1,1 & $15,3-16,1$ & 7,2 & 1,4 \\
\hline Octoblepharum albidum & $20,0-22,5$ & $21,7 \pm 0,2$ & 1,6 & $21,2-22,2$ & 7,5 & $15,0-20,0$ & $17,5 \pm 0,3$ & 2,0 & $17,2-17,8$ & 11,4 & 1,8 \\
\hline O. cocuiense & $20,0-22,5$ & $21,4 \pm 0,2$ & 1,4 & $21,0-21,8$ & 6,7 & $17,5-20,0$ & $18,9 \pm 0,2$ & 1,4 & $18,5-19,3$ & 7,6 & 1,0 \\
\hline
\end{tabular}



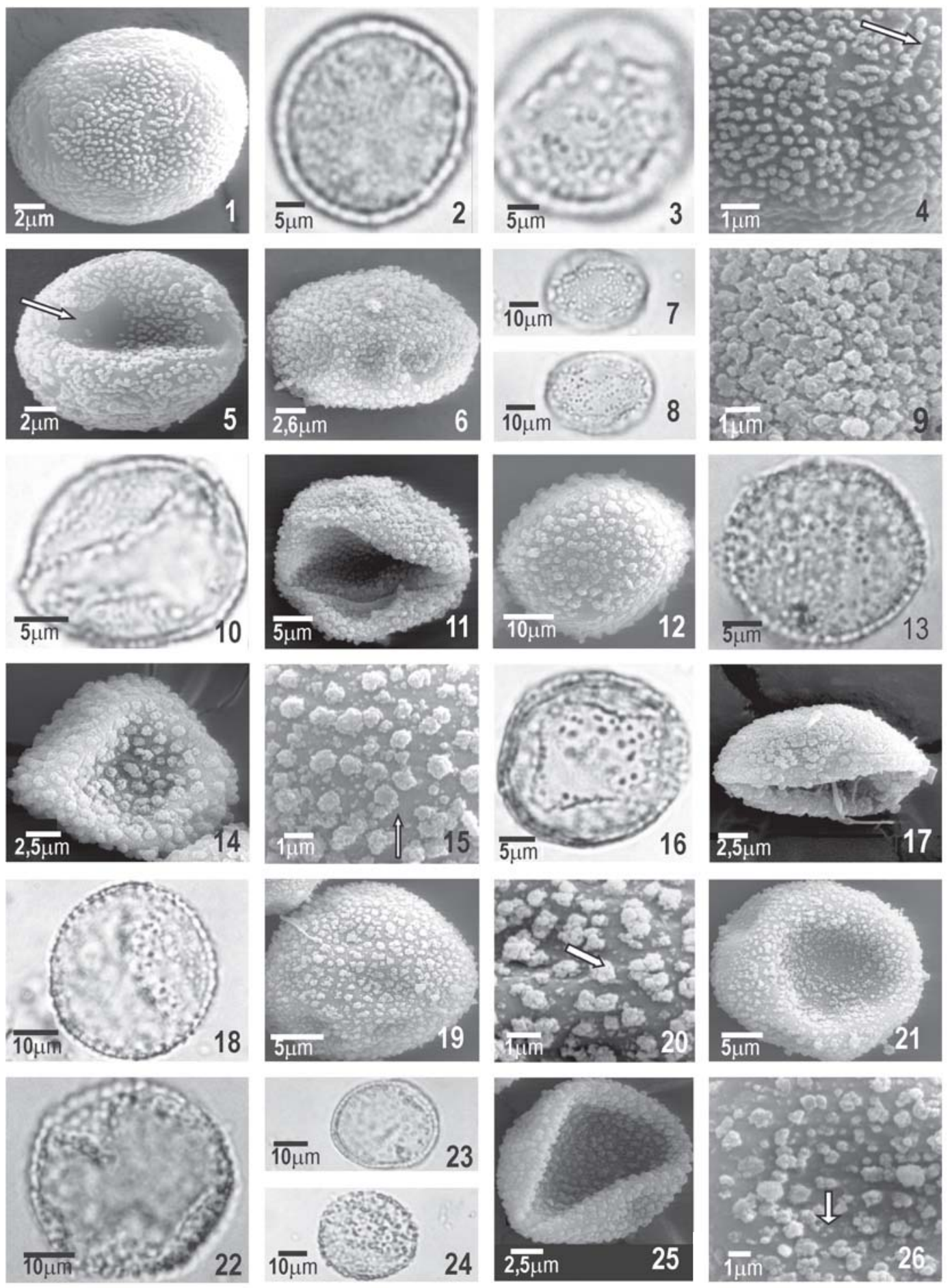

Figuras 1-26. Fotomicrografias e eletromicrografias de esporos de espécies de Leucobryaceae. 1-5. Leucobryum albicans Schwaegr. 1-3. Vista polar distal: 1. Superficie (MEV). 2. Corte óptico (ML). 3. Superfície (ML). 4. Detalhe da superfície (seta, MEV). 5. Vista polar proximal, região apertural (seta, MEV). 6-11. L. clavatum Hampe. 6-8. Vista polar distal: 6. Superfície (MEV). 7-8. Análise LO (ML). 9. Detalhe da superfície (MEV). 10-11. Vista polar proximal: 10. Corte óptico (ML). 11. Região apertural (MEV). 12-15. L. crispum C. Muell. 12-13. Vista polar distal: 12. Superfície (MEV). 13. Superfície (ML). 14. Detalhe da região apertural (MEV). 15. Detalhe da superfície (seta, MEV). 16-17. L. martianum (Hornsch.) Hampe ex C. Muell. 16. Vista polar proximal, região apertural (ML). 17. Vista lateral: superfície (MEV). 18-22. Octoblepharum albidum Hedw. 18-19. Vista polar distal: 18. Corte óptico (ML). 19. Superfície (MEV). 20. Detalhe da superfície granulada (seta), exina psilada (MEV). 21-22. Vista polar proximal: 21. Região apertural (MEV). 22. Região apertural (ML). 23-26. O. cocuiense Mitt. 23-24. Análise LO (ML). 25-26. Vista polar proximal: 25. Região apertural (MEV). 26. Detalhe da perina granulada, presença de nanogrânulos (seta) e exina psilada (MEV). 
apertural subcircular (figuras 21-22). Observa-se uma diminuição dos elementos de ornamentação na região apertural (figura 21).

Octoblepharum cocuiense Mitt. (figuras 23-26) Esporos de tamanho pequeno (tabela 1), perina formada por nanogrânulos e grânulos (figuras 25-26). Os grânulos podem estar isolados ou em grupos (figura 26). Região apertural subtriangular (figura 25).

Todos os materiais de comparação (tabela 2) apresentam morfologia semelhante àquelas observadas no material padrão de cada uma das espécies estudadas. As médias de tamanho dos diâmetros maior e menor de todos os espécimes de comparação estão inseridas nas respectivas faixas de variação de tamanho do material padrão (tabelas 1 e 2), exceto os materiais M.I.M.N. Oliveira-e-Silva 1898 de Leucobryum martianum e M.I.M.N. Oliveira-e-Silva 1557 de Octoblepharum cocuiense que apresentam médias de tamanho

Tabela 2. Dados morfométricos dos esporos acetolisados do material de comparação, em mm (diâmetros maior e menor, $n=30$ e estratos do esporoderma, $n=10)$. ( $\bar{x}=$ média aritmética; $\mathrm{E}=$ espessura do esporoderma).

Table 2. Morphometric data of acetolyzed spores from the comparision specimen, in mm (largest and smallest diameter, $n=30$ and sporoderm strata, $n=10) .(\bar{x}=$ arithmetic mean; $E$ = thickness of sporoderm $)$.

\begin{tabular}{|c|c|c|c|c|c|}
\hline \multirow[t]{2}{*}{ Material } & \multicolumn{2}{|c|}{ Diâmetro maior } & \multicolumn{2}{|c|}{ Diâmetro menor } & \multirow[t]{2}{*}{$\mathrm{E}$} \\
\hline & $\mathrm{X}_{\min }-\mathrm{X}_{\operatorname{máx}}$ & $\bar{x}$ & $\mathrm{X}_{\min }-\mathrm{X}_{\operatorname{máx}}$ & $\bar{x}$ & \\
\hline \multicolumn{6}{|l|}{ Leucobryum albicans } \\
\hline M.I.M.N. Oliveira-e-Silva 1909 & $12,5-17,5$ & 15,7 & $10,10-15,0$ & 13,0 & 1,8 \\
\hline O. Yano \& M.I.M.N. Oliveira-e-Silva 23669 & $15,0-17,5$ & 15,4 & $13,0-15,0$ & 13,0 & 1,8 \\
\hline M.I.M.N. Oliveira-e-Silva 1951 & $15,0-17,5$ & 15,2 & $13,7-15,0$ & 13,5 & 2,2 \\
\hline \multicolumn{6}{|l|}{ L. clavatum } \\
\hline M.I.M.N. Oliveira-e-Silva 2549 & $15,0-20,0$ & 17,2 & $11,2-15,0$ & 13,0 & 1,2 \\
\hline M.I.M.N. Oliveira-e-Silva 3126 & $16,2-18,8$ & 17,1 & $12,5-15,2$ & 13,4 & 1,5 \\
\hline O. Yano \& M.I.M.N. Oliveira-e-Silva 23802 & $16,2-20,0$ & 16,5 & $10,0-15,0$ & 12,6 & 1,2 \\
\hline \multicolumn{6}{|l|}{ L. crispum } \\
\hline O. Yano \& M.I.M.N. Oliveira-e-Silva 1912 & $21,2-25,0$ & 23,5 & $15,0-20,0$ & 20,6 & 2,2 \\
\hline M.I.M.N. Oliveira-e-Silva 2042 & $20,0-22,0$ & 23,5 & $20,0-22,5$ & 20,9 & 2,4 \\
\hline M.I.M.N. Oliveira-e-Silva 2040 & $20,0-23,8$ & 23,0 & $20,0-22,5$ & 20,4 & 1,8 \\
\hline \multicolumn{6}{|l|}{ L. martianum } \\
\hline M.I.M.N. Oliveira-e-Silva 1904 & $18,8-19,4$ & 19,4 & $15,0-17,5$ & 15,9 & 1,4 \\
\hline M.I.M.N. Oliveira-e-Silva 1898 & $20,0-23,4$ & 21,5 & $17,5-20,0$ & 19,2 & 1,2 \\
\hline M.I.M.N. Oliveira-e-Silva 2493 & $16,2-21,2$ & 19,4 & $15,0-20,0$ & 15,6 & 1,2 \\
\hline \multicolumn{6}{|l|}{ Octoblepharum albidum } \\
\hline M.I.M.N. Oliveira-e-Silva 2606 & $21,2-22,5$ & 21,0 & $16,2-20,0$ & 17,2 & 2,0 \\
\hline M.I.M.N. Oliveira-e-Silva 2703 & $20,0-22,5$ & 21,5 & $16,2-17,5$ & 17,4 & 1,8 \\
\hline M.I.M.N. Oliveira-e-Silva 2081 & $20,0-25,0$ & 22,0 & $17,5-20,0$ & 17,0 & 1,8 \\
\hline \multicolumn{6}{|l|}{ O. cocuiense } \\
\hline M.I.M.N. Oliveira-e-Silva 2087 & $18,8-21,2$ & 18,8 & $18,8-20,0$ & 19,0 & 1,2 \\
\hline M.I.M.N. Oliveira-e-Silva 1557 & $20,0-23,8$ & 21,2 & $18,8-22,5$ & 21,8 & 1,2 \\
\hline
\end{tabular}

Figures 1-26. Photomicrographs (LM) and electromicrographs (SEM) of Leucobryaceae species spores. 1-5. Leucobryum albicans Schwaegr. 1-3. Distal polar view: 1. Surface (SEM). 2. Optical section (LM). 3. Surface (LM). 4. Surface detail (arrow, SEM). 5. Proximal polar view, apertural region (arrow, SEM). 6-11. L. clavatum Hampe. 6-8. Distal polar view: 6. Surface (SEM). 7-8. OL analysis (LM). 9. Surface detail (SEM). 10-11. Proximal polar view: 10. Optical section(LM). 11. Apertural region (SEM). 12-15. L. crispum C. Muell. 12-13. Distal polar view: 12. Surface (SEM). 13. Surface (LM). 14. Apertural region detail (SEM). 15. Surface detail (arrow, SEM). 16-17. L. martianum (Hornsch.) Hampe ex C. Muell. 16. Proximal polar view, apertural region (LM). 17. Lateral view: surface (SEM). 18-22. Octoblepharum albidum Hedw. 18-19. Distal polar view: 18. Optical section (LM). 19. Surface (SEM). 20. Granulated surface detail (arrow), psilate exine (SEM). 21-22. Proximal polar view: 21. Apertural region (SEM). 22. Apertural region (LM). 23-26. O. cocuiense Mitt. 23-24. OL analysis (LM). 25-26. Proximal polar view: 25. Apertural region (SEM). 26. Granulated perine, presence of nanogranules (seta) and psilate exine (SEM). 
superiores aos respectivos materiais padrão.

O estudo dos esporos de espécies de Leucobryum e de Octoblepharum demonstrou que os elementos de ornamentação compreendem nanogrânulos ou grânulos em todas as espécies.

As principais diferenças encontradas estão relacionadas à distribuição dos elementos de ornamentação, ao tamanho dos esporos (tabela 1) e à espessura do esporoderma (tabela 2).

No que se refere à ornamentação, as variações na distribuição dos grânulos da perina permitiram a diferenciação das espécies. Leucobryum albicans Schwaegr apresentou grânulos dispostos formando "muros" na parede; em L. clavatum Hampe os grânulos são em grande número em toda a extensão da parede, em algumas regiões estão sobrepostos. Os grânulos em L. crispum C. Muell são maiores e observa-se a presença de nanogrânulos isolados na superfície. No gênero Octoblepharum, os grânulos estão agrupados e observa-se a presença de nanogrânulos dispersos na superfície. Em O. albidum Hedw observa-se uma diminuição no número de grânulos na região apertural.

A Tabela 1 mostra que os coeficientes de variação são superiores a $7 \%$ e variam desde $7,2 \%$ em Leucobryum martianum (Hornsch.) Hampe ex C. Muell a 14,2\% em Leucobryum clavatum, demonstrando uma grande dispersão nas medidas das médias de tamanho destes esporos. Esses caracteres não foram aqui utilizados isoladamente para a caracterização dos esporos, pois as variações ocorridas podem estar relacionadas a diferenças de maturidade dos esporos de uma mesma espécie.

O coeficiente de variação de tamanho encontrado em Leucobryum albicans e L. clavatum é considerado alto. Essa observação está de acordo com os resultados obtidos por Sorsa \& Koponen (1973) quando estudaram os esporos de musgos da família Mniaceae. Os autores concluíram que as maiores diferenças encontradas entre os esporos das 42 espécies examinadas estavam relacionadas ao tamanho, observando que as dimensões dos esporos variavam dentro de um limite relativamente grande e não poderiam ser usados como um caráter isolado para a delimitação de espécies, em consonância com os resultados aqui obtidos.

As variações na distribuição dos grânulos examinados nos exemplares da família Leucobryaceae, aliadas a caracteres de tamanho e simetria, confirmam as observações de McClymont (1955) de que os esporos de musgos não são monotonamente uniformes como haviam sido caracterizados por Knox (1939).
O esporoderma apresenta perina, exina e intina. Esses resultados concordam com a observação de Brown \& Lemmon (1984a) no trabalho de análise da ultraestrutura da esporogênese do musgo Amblystegium riparium Hedw., no qual os autores delimitam as três camadas supracitadas, como as camadas presentes no esporoderma dos esporos dos musgos.

Tal como esperado, a intina foi destruída no processo de acetólise, a perina e a exina são tolerantes a esse processo, desde que sejam alterados o tempo de permanência e a temperatura do banho-maria. Esses dados corroboram as análises realizadas por Neidhart (1979) que reporta a presença da esporopolenina tanto na exina quanto na perina dos musgos, conferindo maior resistência nestes estratos.

Os resultados encontrados caracterizam a família Leucobryaceae como euripalinológica.

Agradecimentos - As autoras são gratas a todos aqueles que colaboraram na execução do presente trabalho, especialmente, à Dra. Olga Yano pelas valiosas sugestões, à Dra. Maria Isabel de Oliveira e Silva pelo empréstimo do material botânico e auxílio com a bibliografia, ao Departamento de Metalurgia da PUC-Rio, na pessoa da Técnica Maria de Fátima Silva Lopes pelo uso do microscópio eletrônico de varredura, à Capes pela bolsa concedida à primeira autora e à Coordenação da Pós-Graduação do Museu Nacional, representada pela Dra. Lygia Dolores Fernandes Ribeiro, pelo apoio.

\section{Referências bibliográficas}

BARTH, O.M. \& MELHEM, T.S. 1988. Glossário Ilustrado de Palinologia. Unicamp, Campinas.

BOROS, A. \& JÁRAI-KOMLÓDI, M. 1975. An Atlas of recent European Moss Spores. Akadámiai Kiadó, Budapest.

BROTHERUS, V.F. 1925. Musci (Laubmoose). In Die Natürlichen Pflanzenfamilien (A. Engler \& K. Prantl, eds.). W. Engelmann, Leipzig, Häfte 2, p.1908-1124.

BROWN, R.C. \& LEMMON, B.E. 1980. Ultrastrucutre of sporogenesis in moss Ditrichum pallidum II. Spore formation. American Journal of Botany 67:918-934.

BROWN, R.C. \& LEMMON, B.E. 1984a. Ultrastructure of sporogenesis in moss Amblystegium riparium II. Spore wall development. Journal of Hattori Laboratory 57:139-152.

BROWN, R.C. \& LEMMON, B.E. 1984b. Spore wall development in Andreaea (Musci: Bryopsida). American Journal of Botany 71:412-420.

BROWN, R.C. \& LEMMON, B.E. 1988. Sporogenesis in Bryophytes. Advances in Bryology 3:159-223.

BROWN, R.C. \& LEMON, B.E. 1991. Sporogenesis in simple land plants. In Pollen and Spores. Patterns and Diversification (S.B. Blackmore \& S. Barnes, eds.). Clarendon Press, Oxford, p.9-24. 
BUCK, W.R. \& GOFFINET, B. 2000. Morphology and classification of mosses. In Bryophyte Biology (J.A. Shaw \& B. Goffinet, eds.). Cambridge University Press, Cambridge, p.71-123.

CASTAÑEDA-PEÑA, R. 1985. Estudio morfológico de las microsporas de ocho species de musgos mexicanos. Anales de la Escuela Nacional de Ciencias Biologicas 29:9-28.

ERDTMAN, G. 1952. Pollen Morphology and Plant Taxonomy. Angiosperms. An Introduction to Palinology I. Almquist \& Wilksells, Stockholm.

ESTEVES, V.G. \& MELHEM, T.S. 2000. Palitaxonomia de espécies brasileiras de Salacia L. (Hippocrateaceae). Revista Brasileira de Botânica 23:425-440.

FRAHM, J.P. \& GRADSTEIN, S.R. 1991 An altitudinal zonation of tropical rain forests using bryophytes. Biogeography 18:669-678.

HIROHAMA, T. 1977. Spore morphology of bryophytes observed by scanning electron microscope, III. Orthotrichaceae. Bulletin of National Science Museum, ser. B (Bot.) 3:113-122, 7pl.

KNOX, E.M. 1939. The spores of Bryophyte compared with those of the carboniferous age. Transactions of the Botanical Society of Edinburgh 32:477-487.

LUIZI-PONZO, A.P. 2001. Palinotaxonomia de Orthotrichinae Fleisch. (Bryales - Bryophyta) do Brasil. Tese de doutorado, Instituto de Biociências da Universidade de São Paulo, São Paulo.

LUIZI-PONZO, A.P. \& BARTH, O.M. 1998. Spore morphollogy of some Bruchiaceae species (Bryophyta) from Brazil. Grana 37:222-227.

LUIZI-PONZO, A.P. \& BARTH, O.M. 1999. Spore morphology of some Dicranaceae species (Bryophyta) from Brazil. Grana 38:42-49.

LUIZI-PONZO, A.P. \& BARTH, O.M. \& LISBOA, R.C.L. 1997. Estudos Palinológicos em Briófitas. In Caxiuanã (P.L.B. Lisboa ed.). Museu Paraense Emílio Goeldi \& MCT/CNPq, Belém, p.305-318.
McCLYMONT, J.W. 1955. Spore studies in the Musci with special reference to the genus Bruchia. The Bryologist 58:287-306.

MOGENSEN, G.S. 1981. The biological significance of morphological characters in Bryophytes: The spore. The Bryologist 84:187-207.

MÜELLER, C. 1843. Ueber die Laubmoosgruppe der Leucophaneen Hampe, Linnaea 17:315-322.

NEIDHART, H.B. 1979. Comparative studies of sporogenesis in Bryophytes. In Bryophyte Systematics (G.C.S. Clarke \& J.G. Duckett, eds.). London, Academy Press. Systematics Association Special Volume. N.14, p.251-280.

OLENSEN, P. \& MOGENSEN, G.S. 1978. Ultrastructure, histochemistry and notes of germination stages of spores in selected mosses. The Bryologist 81:493-516.

OLIVEIRA-E-SILVA, M.I. \& YANO, O. 2000. Musgos de Mangaratiba e Angra dos Reis. Rio de Janeiro, Brasil. Boletim do Instituto de Botânica 14:1137.

PUNT, W., BLACKMORE, S., NILSSON, S. \& LE THOMAS, A. 1999. Glossary of pollen and spore terminology. http:/ /www.biol.ruunl/ palaeo/glossary/glos-int.htm (acesso em 18/04/1999).

SCHIMPER, R.W. 1836-1855. Muscorum chilensium species novas descripsit: Annales dês Sciences Naturelles, Botanique ser. 2, 6:145-149.

SORSA, P. \& KOPONEM, T. 1973. Spore morphology of Mniaceae Mitt. (Bryophyta) and its taxonomic significance. Annales Botanici Fennici 10:187-200.

VITT, D.H. 1984. Classification of Bryopsida. In New Manual of Bryology (R.M. Schuster, ed.). The Hattori Botanical Laboratory 2:696-759.

WODEHOUSE, R.P. 1935. Pollen Grains. Their structure, identification and significance in science and medicine. McGraw-Hill Book Co., New York.

YANO, O. 1975. Leucobryaceae (Musci) do Estado de São Paulo. Dissertação de mestrado, Escola Paulista de Medicina, São Paulo.

YANO, O. 1996. A checklist of Brazilian bryophytes. Boletim do Instituto de Botânica 10:47-232. 